\title{
Variations in administration of Covid-19 vaccine during last 20 weeks at a vaccination center of Agra, Uttar Pradesh
}

Shailendra Singh Chaudhary ${ }^{1}$, Manisha Madhukar Nagargoje ${ }^{2}$, Himalaya Singh ${ }^{3}$, Kunver Viresh Singh ${ }^{4}$, Rinu Kumar $^{5}$, Waqaar Sayeed ${ }^{6}$

${ }^{1}$ Associate Professor, Department of Social and Preventive Medicine, Sarojini Naidu Medical College, Agra, Uttar Pradesh; ${ }^{2}$ Assistant Professor, Department of Social and Preventive Medicine, Sarojini Naidu Medical College, Agra, Uttar Pradesh; ${ }^{3}$ Epidemiologist cum Assistant Professor, Department of Social and Preventive Medicine, Sarojini Naidu Medical College, Agra, Uttar Pradesh; ${ }^{4}$ Assistant Professor, Department of Social and Preventive Medicine, Sarojini Naidu Medical College, Agra, Uttar Pradesh; ${ }^{5} J u n i o r$ Resident, Department of Social and Preventive Medicine, Sarojini Naidu Medical College, Agra, Uttar Pradesh; ${ }^{6}$ Junior Resident, Department of Social and Preventive Medicine, Sarojini Naidu Medical College, Agra, Uttar Pradesh

\begin{tabular}{|c|c|c|c|c|c|c|c|}
\hline Abstract & Introduction & Methodology & Results & Conclusion & References & Citation & Tables / Figures \\
\hline
\end{tabular}

\section{Corresponding Author}

Dr Kunver Viresh Singh, Assistant Professor, Department of Social and Preventive Medicine, Sarojini Naidu Medical College, Agra - 282002

E Mail ID: drshailen321@yahoo.co.in

\section{Citation}

Chaudhary SS, Nagargoje MM, Singh H, Singh KV, Kumar R, Sayeed W. Variations in administration of Covid-19 vaccine during last 20 weeks at a vaccination center of Agra, Uttar Pradesh. Indian J Comm Health. 2021;33(2):337-343. https://doi.org/10.47203/IJCH.2021.v33i02.020

Source of Funding: Nil Conflict of Interest: None declared

\section{Article Cycle}

Received: 12/05/2021; Revision: 30/05/2021; Accepted: 16/06/2021; Published: 30/06/2021

This work is licensed under a Creative Commons Attribution 4.0 International License.

\section{Abstract}

Background: Covid-19 pandemic has created havoc around the world and vaccination is an effective tool against this demon. Study of variations in administration of daily doses of Covid-19 vaccine at vaccination centre can help in better resource management. Material \& methods: This record based descriptive study was conducted among beneficiaries of Covid-19 vaccination at S. N. Medical College, Agra. Record of administration of daily doses and vaccine wastage, vaccination of different groups of beneficiaries and occurrence of various national and local events related to vaccination was also analyzed. Result: A total of 33,571 doses of any Covid-19 vaccine (Covaxin/Covishield) were administered during past 88 working days in 20 weeks expanded over 5 months. On an average 165 doses were administered every day by each team and overall vaccine wastage was $0.85 \%$. Maximum average of 199 doses per day by each team was observed on Mondays. There are many peaks and drops in administration of daily doses of Covid-19 vaccine at our center which can be attributed to either some national or local events related to supply of vaccine at our center, addition of a new group of beneficiary, any news or political controversy related to Covid-19 vaccination etc. Conclusion: Understanding of spikes and drops in the curve of daily/weekly administration of Covid-19 vaccine, in view of occurrence of various national and local events related to vaccination, can help in better formulation of strategies specially related to human resource allocation for success of National Covid-19 vaccination program.

\section{Keywords}

SARS CoV-2, COVID-19 vaccination, Covid-19 vaccine, Vaccine wastage, COVID-19 vaccination center (CVC)

\section{Introduction}

Since the beginning of the COVID-19 pandemic in 2020, more than 3.5 million lives have been lost and the global society has been disrupted in an unprecedented manner(1). After trying many therapeutic modalities with partial or no success, prevention seems to be a better bet to halt the rapid progression of the pandemic. World Health Organization (WHO) recommended nonpharmaceutical preventive strategies like social distancing, regular use of facial mask, frequent hand sanitization and travel restrictions are successfully used by 
various countries for protection of their citizen against Covid-19 infection(2). In view of current scenario, many countries and pharmaceutical companies have galvanized the development of a number of vaccines that have received emergency use authorization from regulatory bodies of various countries(3).

Two such vaccines were granted emergency use authorization by the Central Drugs Standard Control Organization (CDSCO) in India, Covishield ${ }^{\circledR}$ (AstraZeneca's vaccine manufactured by Serum Institute of India) and Covaxin $^{\circledR}$ (manufactured by Bharat Biotech Limited) in January 2021(4). Third vaccine Sputnik - V has also been granted EUA in the month of April 2021(4).

Covid-19 vaccination in India as well as in our institution was started on January 16, 2021. Various groups like Health Care Workers (HCWs), Front Line Workers (FLWs), person's aged 60 years \& above, $45-59$ years \& above and 18-44 year were covered in phased manner(5).

Since the start of Covid-19 vaccination in India, questions are frequently raised regarding ability of government of India to protect all its 1.3 billion citizen through vaccination by the end of this year $(6,7,8)$. On one hand, government is leaving no stone unturned to meet this gigantic demand but on the other hand, judicious use of available vaccine doses is need of the hour as vaccine wastage has emerged as one of the biggest challenge for most state governments of India. Inadequate training of vaccinators in drawing vaccine from multi-dose vials and lack of detailed planning at vaccination sites are identified as two key gaps that have led to vaccine wastage levels in some of these states above the national average of $6.5 \%(9,10)$.

Though Covid-19 vaccination is running successfully at our institution, a need was felt to analyze the variations in daily vaccination and vaccine wastage for better resource management at our center.

\section{Aim \& Objective}

To analyze variations in administration of COVID-19 vaccine and to recommend suitable suggestions based on the study findings.

\section{Material \& Methods}

Type of study: This is a record based descriptive type of study, Study population: The study was conducted among beneficiaries of Covid-19 vaccination, Site of study: Covid19 Vaccination Center (CVC) of S. N. Medical College, Agra was the site of study, Duration of study: The data collected over 5 months between January to May, 2021 was used for this study, Sample size calculation: This is a record based study and data of all the beneficiaries attending Covid-19 vaccination center (CVC) at S. N. Medical College, Agra over the stipulated time-frame was taken into account for purpose of this study, Methodology: The present study is a record based descriptive study. Covid-19 vaccination in India as well as in our institution was started on January 16, 2021. In phase one, vaccination of the most priority group i.e. health-care workers (HCWs) was started initially which later on expanded to include front line workers (FLWs) in the month of February. Later on, vaccination of individuals aged 45 years and above was started in phase two in March 2021 while vaccination of 18-44 year old persons was started in third phase at the start of month of May. In the beginning, 4 teams were formed in our institution by which vaccination was done only once a week but gradually vaccination days were increased to 2, 3, 4 and 5 days a week. By the month of March, vaccination days were increased to 6 per week while number of teams was reduced to 2. Each Covid-19 vaccination team originally constituted by Chief Medical Officer (CMO), Agra has 6 members: vaccinator officer-1 (security personal), vaccinator officer-2 (verifier), vaccinator officer-3 (mobilizer), vaccinator officer-4 (home guard), vaccinator and additional vaccinator (ANM/GNM). Additionally, nodal officers, security guards, computer operators, classIV staffs and cleaning staffs were also appointed by college administration vis-à-vis work load of daily vaccination.

The COVID-19 Vaccine Intelligence Network (Co-WIN) system, a digital platform was used to track the enlisted beneficiaries for vaccination and COVID-19 vaccines on a real-time basis. At the vaccination site, only pre-registered beneficiaries were vaccinated as per the prioritization, and there was no provision for on-the-spot registrations(11). With roll out of second phase of vaccination, CoWIN 2.0 portal was launched enabling eligible beneficiaries to self-register and book a slot in addition to on-the-spot registration at CVC(12).

During the last 20 weeks, beneficiaries were given either Covaxin or Covishield at our CVC ensuring second dose of same brand of vaccine as first dose. Though both brands of vaccines were supplied to us at different times, Covaxin was available at our CVC during most of the period of study. In-fact, our CVC is locally well known for administration of Covaxin to vaccine beneficiaries. Both Covaxin and Covishield is given in two doses i.e. first and second dose keeping interval of minimum 4 weeks between 2 doses. Interval between 2 doses of Covishield has now increased to 12-16 weeks while interval between 2 doses of Covaxin is still 4-6 weeks. Record of daily vaccination at our CVC was maintained electronically since the beginning. Factors like late supply of vaccine in the morning, glitches in start of a session at CoWIN app, absence/day-off of a vaccinator/verifier were also observed but their effect on day to day variations in vaccination was mostly mitigated by appropriate early interventions and thus probably have no effect in weekly and monthly variations in Covid-19 vaccination.

Various national and local events related to vaccination like addition of a new group of beneficiary, availability/absence of a particular type of vaccine at our CVC, formidable second wave of Covid-19 pandemic in Agra city, occurrence of festivals like Holi and Nav-ratri 
etc. were also recorded. An analysis of weekly variations was done in respect to such Covid-19 vaccination related events.

Despite best of the efforts, vaccine wastage happens in all programs chiefly during transportation, storage and at vaccination centers. Covaxin in India was introduced as 20-dose vial while Covishield was launched as 10-dose vial. Later-on, 20-dose vial was replaced by 10 -dose vial of Covaxin to minimize vaccine wastage. A vial of COVID-19 vaccine has to be used within a fixed time period (4 hours) after opening it. If 10 people are not available to get inoculated during the time period, then the left over doses is wasted.

All the data thus collected was entered in Microsoft Excel daily which was later on clubbed to analyze weekly and monthly trends in Covid-19 vaccination. As for other descriptive studies, percentages and proportion were used to analyze the data. Results were compared with available studies and inferences were drawn based on the findings of the study.

\section{Results}

A total of 33,571 doses were inoculated during past 88 working days in 20 weeks expanded over 5 months. (Table 1) shows distribution of Covid-19 vaccine type (Covaxin and Covishield) and dose (first and second) in different population sub-groups. Accordingly, 5312 doses were administered to HCWs, 3485 to FLWs, 7531 to person above sixty years of age, 11007 doses to persons aged 4559 years and 6236 doses were given to persons aged 18 44 years. Of the total, $65 \%$ were first doses while $35 \%$ were second doses. Three fourth of vaccine doses administered at our CVC were Covaxin while one fourth were Covishield. Reason for usage of a particular brand of vaccine is mostly administrative and is related to availability/supply of vaccine at that time (Table 2).

\section{Daily variation in Covid-19 vaccination}

It was observed that minimum 41 doses of Covid-19 vaccine were administered on January 16th 2021 (first day of vaccination) while maximum 850 doses of doses were administered on May 10th 2021 (first day of vaccination among persons below 45 years of age). (Table 2) shows that a total of 33,571 doses of Covid-19 vaccines were inoculated during past 88 working days. In the start; 4 teams were formed for Covid-19 vaccination (except for day 1 when only one team was assigned for vaccination) but number of teams was reduced to 2 in later stage. Total team-days of Covid-19 vaccination were 203 while daily average of vaccination per team was calculated to be 165 doses per day by each team. There are many peaks and drops in daily coverage of Covid-19 vaccination at our center which is evident from the (Figure 1).

Days-wise distribution of Covid-19 vaccination shows that maximum vaccine doses were given on Fridays followed by Mondays and Thursdays (Table 3 ). This do not represent true picture of daily vaccination as in first phase only Fridays and Thursdays were chosen for Covid-19 vaccination. When calculation of work load was done it was observed that maximum 199 doses (Figure 2) were administered on an average by each team on Mondays while only 132 doses were administered on Thursdays and 130 were administered on Sunday (which was the only working Sunday till date and was incidentally the first day of 4-days long Tika Utsav).

\section{Weekly variations in Covid-19 vaccination}

In the beginning; vaccination was done only once every week which was later on increased to 2-3 days per week and later on maximized to 6 days a week in the month of March except for 3rd week of April where vaccination sessions were also held on Sunday to celebrate Tika-Utsav (Vaccine Festival). Graph 3 shows that weekly Covid-19 vaccine coverage at S. N. Medical College, Agra was increased from 41 in first week to 3,440 at the end of the 20th week. A total of 33,571 doses were inoculated during past 20 weeks with an average of 1679 doses per week (Table 2).

Weekly Covid-19 vaccination graph shows four distinct peaks (Figure 3 ).

First peak at 8th week of vaccination corresponds to rising public confidence in vaccine as Prime Minister himself took first dose of indigenous vaccine Covaxin (Table 2). Facility of on the spot registration of individuals aged more than 60 years and individuals between $45-59$ years with specified co-morbidities in addition to abolition of previous strategy of vaccination from pre-fixed list of beneficiaries only was also started in this week. COWIN 2.0 app was launched at the start of second phase of vaccination drive resulting in quicker vaccination process with inoculation of much larger number of doses by each team per day.

Second peak at 10th week was probably attributed to strategy of vaccination on 6 days a week (except Sunday) even on Gazetted Holidays. Extensive electronic and print media coverage of vaccine administration to local prominent public figures like city Mayor, state Minister, elected Members of Legislative Assembly (MLAs) etc. at our CVC during 9th and 10th week also led to rush of common public to our center for Covid-19 vaccination.

Third peak at 13th week coincide well with administration of second dose of vaccine to Prime Minister and start of vaccination of all individuals aged 45 years and above thus removing necessity of co-morbidity in this group as a criteria of eligibility for Covid-19 vaccination.

Fourth peak at 19th week has some interesting finding as availability of Covaxin seems to improve the vaccination numbers over the previous week which incidentally was the first week of Covid-19 vaccination for 18-44 years age group in Agra district.

Monthly variation and vaccine wastage in Covid-19 vaccination

Total number of doses of Covid-19 vaccine administered at our institution significantly increased from 765 in 
month of January to 13628 in May (till 30th May 2021).

Maximum 374\% increase was observed between month of February (1922 doses) and March (7194 doses) which can be attributed to drastic expansion of beneficiary base (with addition of people aged 60 plus and 45 plus with specified co-morbidities) and increase in number of vaccination days from 3 to 6 in the month of March.

With increase in number of vaccine doses administered per month, wastage of vaccine decreased sharply ( 4). Overall 289 doses of Covid-19 vaccines were wasted among 33860 doses utilized by us leading to less than one percentage vaccine wastage at our institution. In the beginning, vaccine wastage was quite high i.e. $4.37 \%$ and $3.42 \%$ in month of January and February respectively which was decreased to less than two percentages in the month of March. Drastic decrease in vaccine wastage to a level of $0.18 \%$ and $0.16 \%$ was observed in the month of April and May respectively (Table 4).

Vaccine wastage was nil from April 2nd to May 8th but again rose due to re-introduction of pre-fixed list of beneficiaries in the age group of 18-44 years. After 7 days of hitch, we were again able to achieve zero wastage by ensuring 10 persons before opening of a vaccine vial to ensure no wastage at all.

\section{Discussion}

Overall first dose outnumbered second dose which might be due to recent surge in pace of vaccination in different groups, recent increase in gap between 2 doses of vaccine (specially Covishield) and omission of second dose by many due to side effect, forgetfulness or any disease/Covid-19 after first dose. Difference in vaccine type among various groups is mostly due to administrative reason like availability of vaccine rather than their choice. Lately, many persons chose our center for Covid-19 vaccination as Covaxin was mostly available at our place during entire period of the study.

Though increase in administration of monthly, weekly and daily doses of covid-19 vaccine seems significant over the period, this curve is far from smooth. There are many peaks and drops in administration of daily doses of Covid19 vaccine at our center which can be attributed to either some national or local events related to supply of vaccine, addition of new group of beneficiary, political controversy or news related to Covid-19 vaccination etc. as mentioned in the table 2. Relative scarcity of a particular brand of vaccine like for example absence of Covaxin at our CVC for first dose during 14th and 20th week of vaccination was associated with a sharp decrease in number of average weekly vaccine doses administered at our CVC.

Apart from that a lower vaccine dose administration on Thursday can be attributed to deep rooted myth in local society where a person is not supposed to visit any hospital on a Thursday out of fear of repetitive frequent visits to the hospital. Similarly, lowest turnout on Sunday depicts common public perception of a non-working day.
CVC staff also need one day break at the end of the week to avoid burnout due to continuous work for more than 8 hours each day.

Overall vaccine wastage of Covishield at our CVC was $1.77 \%$ while wastage of Covaxin was $0.54 \%$ which in well below the district average of $2.77 \%$ for Covishield and $1.17 \%$ for Covaxin(21). In the beginning, vaccine wastage was quite high i.e. $4.37 \%$ and $3.42 \%$ in month of January and February respectively as a pre-fixed list of beneficiaries for Covid-19 vaccination were provided to us at the start of each day and we had no authority to add potential beneficiary to minimize wastage at our level. Later on, with start of on spot registration of persons above 60 years and 45-59 years with co-morbidity, wastage of vaccine was decreased to less than two percentages in the month of March. Drastic decrease in vaccine wastage to a level of $0.18 \%$ and $0.16 \%$ was observed in the month of April and May respectively partially due to immense administrative pressure in addition of full flexibility in enrollment of beneficiaries at our level.

\section{Conclusion}

Though Covid-19 vaccination rate at our center has steadily increased over the period of time, the curve is not smooth. Spikes and drops in daily/weekly administration of Covid-19 vaccine can help in formulation of strategy for success of program. Regular supply of a particular brand of vaccine at a designated CVC should be ensured otherwise daily/weekly vaccine dose administration decreases whenever people don't get the specific vaccine which they are supposed to get at that center routinely. Extra man power like security staff, vaccinator etc. can be deployed in view of increase in expected number of beneficiaries especially on Mondays and after announcement of start of vaccination for some special group of people. Grant of leave to vaccination staff can also be better managed keeping in view the expected vaccination load on different days of the week. Addition of a special service like vaccination camp or special group invitation can be restricted to Thursdays and Fridays only. Vaccine wastage can be reduced by ensuring 10 persons before opening of a vaccine vial. Alternatively, reregistration of those who had missed their pre-booked slot on previous day can be allowed during next session to minimize wastage without troubles of re-booking.

\section{Relevance of the study}

Covid-19 vaccination is at highest priority of government of India and optimization of available resources is the key for success of program. The present study highlights need of microplanning for better outcome at the CVC.

\section{Authors Contribution}

All authors contributed equally. 


\section{Acknowledgement}

Dr. Sanjay Kala, Principal, S. N. College, Agra and Dr. S. K. Misra, Professor and Head, Department of S.P.M. for giving this team an opportunity to become a part of this historical nation-wide movement of Covid-19 vaccination and providing all the necessary resource and man-power from college and department for smooth functioning of daily vaccination activities. We are thankful to all team members of Covid-19 vaccination

\section{References}

1. COVID-19 CORONAVIRUS PANDEMIC statistics. Available from https://www.worldometers.info/coronavirus/

2. Coronavirus disease (COVID-19) advice for the public by World Health Organization. Available from https://www.who.int/emergencies/diseases/novel-coronavirus2019/advice-for-public [Accessed 25/06/2021]

3. Covid-19 vaccines by World Health Organization. Available from https://www.who.int/emergencies/diseases/novel-coronavirus2019/covid-19-vaccines [Accessed 25/06/2021]

4. Ministry of Health and Family Welfare, Government of India. Frequently asked questions - About the vaccine. Available from https://www.mohfw.gov.in/covid vaccination/vaccination/faqs.h tml\#about-the-vaccine [Accessed 25/06/2021]

5. Liberalised Pricing and Accelerated National Covid-19 Vaccination Strategy. 21/4/2021 Available from https://www.mohfw.gov.in/pdf/LiberalisedPricingandAccelerated NationalCovid19VaccinationStrategy2042021.pdf

6. COVID-19 vaccine data analysis: At current rate of $2.2 \mathrm{mn}$ doses per day, India can only cover $30 \%$ population by end-2021. Tejal Kanitkar. Published on April 25, 2021 16:25:31 IST. Available from https://www.firstpost.com/india/covid-19-vaccine-data-analysisat-current-rate-of-2-2mn-doses-per-day-india-can-only-cover-30population-by-end-2021-9562811.html

7. Coronavirus | India can expect around 1.3 billion doses of vaccines, says epidemiologist Chandrakant Lahariya. Published on May 21, 2021 18:08 IST. Available from: https://www.thehindu.com/news/national/coronavirus-indiaought-to-realistically-expect-around-13-billion-doses-of-vaccinessays-epidemiologist-chandrakant-lahariya/article34615591.ece

8. Divya J Shekhar, Manu Balachandran. Big story: How will India vaccinate 1.3 billion people? 14 min read. Published on Nov 27, 2020 05:00:49 PM IST. Available from: https://www.forbesindia.com/article/take-one-big-story-of-theday/big-story-how-will-india-vaccinate-13-billion-people/64527/1

9. Shortage and wastage: On cutting vaccine wastage. Editorial published on APRIL 29, 2021 00:02 IST. Available from https://www.thehindu.com/opinion/editorial/shortage-andwastage-the-hindu-editorial-on-cutting-covid-19-vaccinewastage/article34433580.ece

10. Centre tracks Covid-19 vaccine wastage: Lack of trained personnel, planning at site level. Written by Kaunain Sheriff M. Updated on March 19, 2021 7:16:30 am. Available from
[Variations in...] | Chaudhary SS et al https://indianexpress.com/article/india/covid-vaccine-wastagecentre-7234943/

11. Ministry of Health and Family Welfare. Covid-19 vaccine: Operational Guidelines. Available from https://www.mohfw.gov.in/pdf/COVID19VaccineOG111Chapter1 6.pdf [Accessed 25/06/2021]

12. CoWIN 2.0 app's soft launch on March 1. The Indian Express. Published on 27th February 2021 08:12 AM. Available from https://www.newindianexpress.com/states/telangana/2021/feb/ 27/cowin-20-apps-soft-launch-on-march-1-2269615.html

13. R. Ramachandran. Politics drives India's fight against the COVID-19 pandemic even as questions over DCGI's approvals for vaccines persist. Cover story in Frontline Magazine. Print edition: January 29, 2021. Available from https://frontline.thehindu.com/coverstory/covid19-vaccine-approval-process-proves-politics-is-drivingindia-s-war-against-the-coronaviruspandemic/article33539431.ece

14. Bharat Biotech: Controversy swirls around India's homegrown Covid-19 vaccine. The Economic Times. 6th March 2021. Available from https://economictimes.indiatimes.com/news/politics-andnation/controversy-swirls-around-indias-homegrown-covid-19vaccine/articleshow/80661723.cms?utm source=contentofintere st\&utm medium=text\&utm campaign=cppst

15. COVID vaccine rollout faces political controversies in India. News program on Times NOW channel. 5 January 2021 05:48 IST. Available from: https://www.timesnownews.com/videos/timesnow/india/covid-vaccine-rollout-faces-political-controversies-inindia/85657

16. Omar Rashid. Moradabad hospital ward boy dies after COVID-19 vaccination, officials say death due to heart disease not vaccine. The Hindu. LUCKNOW, JANUARY 18, 2021 12:37 IST. Available from: $\quad$ https://www.thehindu.com/news/national/otherstates/moradabad-hospital-ward-boy-dies-after-covid-19vaccination-officials-say-death-due-to-heart-disease-notvaccine/article33599210.ece

17. Healthcare worker in Telangana dies 4 days after getting vaccinated. The Hindu Net Desk. January 24, 2021 23:05 IST. https://www.thehindu.com/news/national/coronavirus-january24-2021-live-updates/article33648089.ece

18. The Indian Express. PM Narendra Modi takes first dose of Covaxin at Delhi's AllMS. Express Web Desk. Updated on March 1, 2021 9:21:03 am. Available from https://indianexpress.com/article/india/pm-narendra-modicoronavirus-vaccine-7208991/

19. Modi gets 2 nd dose of COVID-19 vaccine at AlIMS. By Bindu Shajan Perappadan. APRIL 08, 2021 08:23 IST. Available from https://www.thehindu.com/news/national/pm-modi-gets-2nddose-of-covid-19-vaccine-at-aiims/article34268984.ece

20. Statistics: New cases and deaths. Available from https://github.com/CSSEGISandData/COVID-19 [Accessed 25/06/2021]

21. Dainik jagran. वैक्सीन की बर्बादी को रोकने का प्रयास. 27 May 2021. Available from https://www.jagran.com/uttarpradesh/agra-city-efforts-of-save-covid-vaccine-no-wastage-inagra-21683072.html

\section{Tables}

TABLE 1 COVID-19 VACCINATION IN DIFFERENT GROUPS OF POPULATION

\begin{tabular}{|lccccc|}
\hline Group & \multicolumn{2}{c}{ Dose } & \multicolumn{2}{c|}{ Vaccine } \\
\hline Health Care Workers (HCWs) & First & Second & Covishield & Covaxin \\
\hline Front Line Workers (FLWs) & 3003 & 2309 & 2646 & 2666 & 5312 \\
\hline Persons aged 60 years \& above & 1450 & 2035 & 818 & 2667 & 3485 \\
\hline Persons aged 45-59 years & 4362 & 3169 & 211 & 7320 & 7531 \\
\hline Persons aged 18-44 years & 6759 & 4248 & 723 & 10284 & 11007 \\
\hline Total & 6236 & 0 & 4040 & 2196 & 6236 \\
\hline
\end{tabular}


TABLE 2 WEEKLY COVID-19 VACCINATION AND SOME SIGNIFICANT NATIONAL/LOCAL EVENTS OF THE WEEK

\begin{tabular}{|c|c|c|c|c|c|}
\hline Week & $\begin{array}{l}\text { Total doses } \\
\text { given in } \\
\text { week }\end{array}$ & $\begin{array}{l}\text { Vaccina- } \\
\text { tion days } \\
\text { in week }\end{array}$ & $\begin{array}{l}\text { Total team- } \\
\text { days in } \\
\text { week* }\end{array}$ & $\begin{array}{l}\text { Daily average } \\
\text { per team in } \\
\text { week }\end{array}$ & Significant national/local events in the week \\
\hline 1 & 41 & 1 & 1 & 41 & $\begin{array}{l}\text { Pre-fixed list of beneficiary }{ }^{(11)}, \text { Apprehension of new } \\
\text { vaccine, Political controversies }\end{array}$ \\
\hline 2 & 118 & 1 & 4 & 30 & $\begin{array}{l}\text { Pre-fixed list with once a week vaccination, Rumours } \\
\text { of serious side effects or death after vaccination }\end{array}$ \\
\hline 3 & 606 & 2 & 8 & 76 & $\begin{array}{l}\text { Rising confidence in vaccine and two days per week } \\
\text { vaccination }\end{array}$ \\
\hline 4 & 431 & 2 & 8 & 54 & $\begin{array}{l}\text { Vaccination of HCWs only with some repetitions in the } \\
\text { list }\end{array}$ \\
\hline 5 & 490 & 2 & 8 & 61 & Vaccination of FLWs at our CVC \\
\hline 6 & 438 & 3 & 11 & 40 & $\begin{array}{l}\text { Many repetitions in beneficiaries list, Mostly second } \\
\text { dose for HCWs }\end{array}$ \\
\hline 7 & 563 & 3 & 12 & 47 & $\begin{array}{l}\text { Many repetitions in beneficiaries list, Mostly second } \\
\text { dose for HCWs }\end{array}$ \\
\hline 8 & 1744 & 4 & 11 & 159 & $\begin{array}{l}\text { Rising confidence in vaccine, PM took first dose of } \\
\text { Covaxin }^{(18)} \text {, No pre-fixed list of beneficiaries, } \\
\text { vaccination for } 60 \text { plus and } 45 \text { plus comorbid started, } \\
\text { User friendly COWIN } 2.0 \text { app launched }{ }^{(12)}\end{array}$ \\
\hline 9 & 1398 & 5 & 10 & 140 & $\begin{array}{l}\text { Number of vaccination teams reduced from } 4 \text { to } 2,6 \\
\text { days a week vaccination except } \mathrm{GH}\end{array}$ \\
\hline 10 & 2262 & 6 & 12 & 189 & $\begin{array}{l}6 \text { days a week vaccination even on } \mathrm{GH} \text {, Local public } \\
\text { figures got vaccinated }\end{array}$ \\
\hline 11 & 1582 & 6 & 12 & 132 & Holi-dip before festive season \\
\hline 12 & 1570 & 4 & 8 & 196 & $\begin{array}{l}\text { Announcement of vaccination for all } 45 \text { plus } \\
\text { individuals }\end{array}$ \\
\hline 13 & 3180 & 6 & 12 & 265 & $\begin{array}{l}\text { PM took second dose of vaccine }{ }^{(19)} \text {, very high turnout } \\
\text { of } 45 \text { plus individuals }\end{array}$ \\
\hline 14 & 2150 & 7 & 14 & 154 & Tika-Utsav, Nav-ratri festival, No Covaxin for first dose \\
\hline 15 & 1930 & 6 & 12 & 161 & Nav-ratri festival (9-days fast) \\
\hline 16 & 1610 & 6 & 12 & 134 & $\begin{array}{l}\text { Lock-down started, Second wave of Covid-19 } \\
\text { pandemic at its peak in Uttar Pradesh }\end{array}$ \\
\hline 17 & 1970 & 6 & 12 & 164 & Covid-19 cases started to fall from peak \\
\hline 18 & 3882 & 6 & 12 & 324 & $\begin{array}{l}\text { Vaccination for } 18-44 \text { years started in Agra but no } \\
\text { Covaxin for them }\end{array}$ \\
\hline 19 & 4166 & 6 & 12 & 347 & $\begin{array}{l}\text { Covaxin for first dose for people of } 18-44 \text { years as well } \\
\text { as } 45 \text { plus group }\end{array}$ \\
\hline 20 & 3440 & 6 & 12 & 287 & No Covaxin for first dose in any group \\
\hline Total & 33571 & 88 & 203 & 165 & \\
\hline
\end{tabular}

TABLE 3 DAYS-WISE COVID-19 VACCINATION

\begin{tabular}{|lccc|}
\hline Day & Total doses administered & Number of days & Doses per day \\
\hline Monday & 6180 & 31 & 199 \\
\hline Tuesday & 4278 & 22 & 194 \\
\hline Wednesday & 4234 & 23 & 184 \\
\hline Thursday & 6064 & 46 & 132 \\
\hline Friday & 7327 & 52 & 141 \\
\hline Saturday & 5228 & 27 & 194 \\
\hline Sunday & 260 & 2 & 130 \\
\hline
\end{tabular}


TABLE 4 MONTH-WISE WASTAGE OF DIFFERENT COVID-19 VACCINES (IN PERCENTAGES)

\begin{tabular}{|lcccc|}
\hline Month & Covishield & Covaxin-20 dose vial & Covaxin-10 dose vial & Total \\
\hline January & 4.37 & N.A. & N.A. & 4.37 \\
\hline February & 3.89 & 2 & N.A. & 3.42 \\
\hline March & 3.17 & 1.74 & N.A. & 1.99 \\
\hline April & 0.1 & 3.54 & 0 & 0.18 \\
\hline May & 0.44 & N.A. & 0.04 & 0.16 \\
\hline Overall & 1.77 & 1.88 & 0.02 & 0.85 \\
\hline N.A. - Not available & & & & \\
\hline
\end{tabular}

\section{Figures}

\section{FIGURE 1 DAILY COVID-19 VACCINATION}

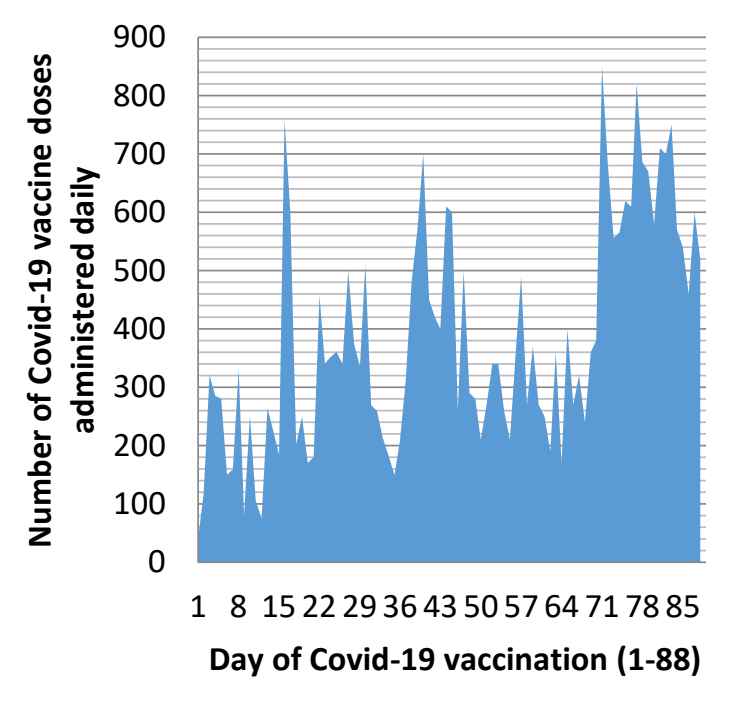

FIGURE 2 DAY-WISE COVID-19 VACCINATION

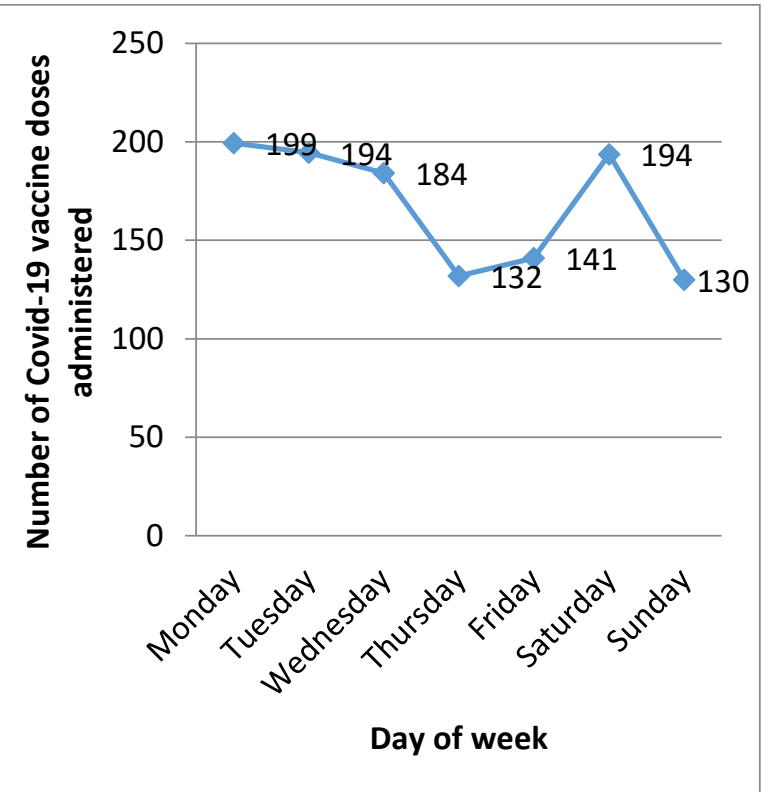

FIGURE 3 WEEKLY COVID-19 VACCINATION

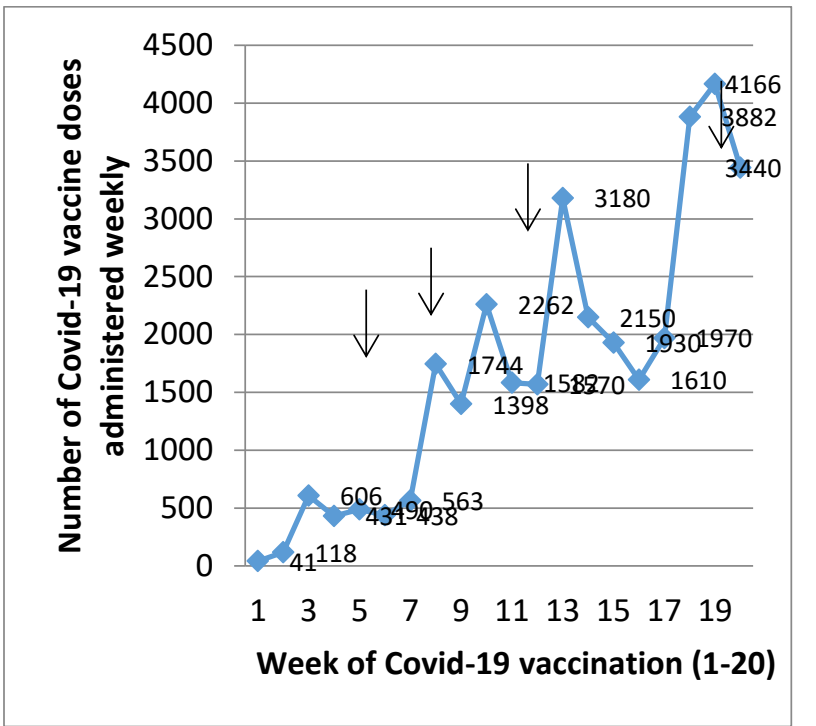

FIGURE 4 MONTH-WISE COVID-19 VACCINATION AND VACCINE WASTAGE (\%)

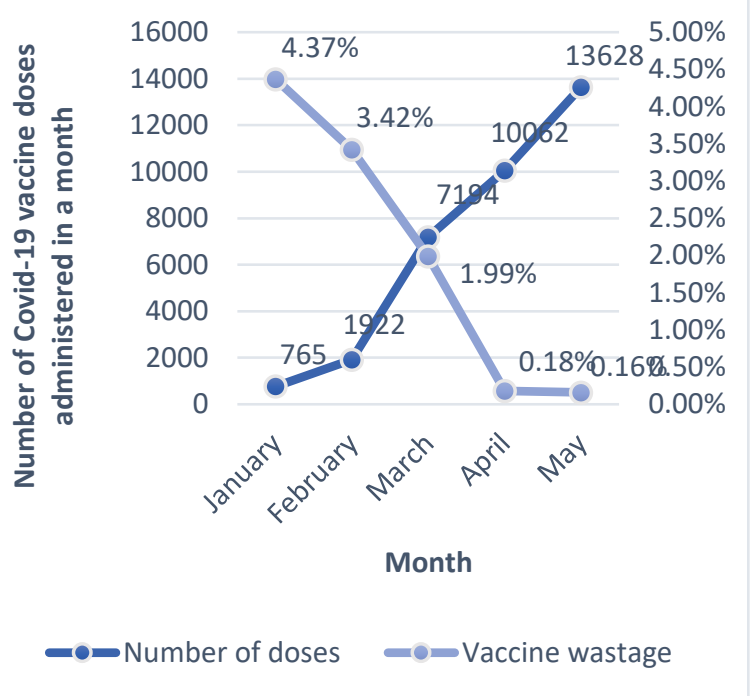

